\title{
Estimation of type II truncated Fréchet inverse exponential distribution under censored data
}

\author{
Maha A. Aldahlan \\ Department of Statistics, College of Science, University of Jeddah, Jeddah, Saudi Arabia.
}

\begin{abstract}
This paper proposes a new probability distribution called as type II truncated Fréchet inverse exponential distribution. Fundamental statistical properties like moments, incomplete moments, and quantile function of the proposed model are studied. The estimation of the parameter of the new model is approached by maximum likelihood method based on complete and censored samples. Asymptotic confidence interval of model parameters is investigated. We assess the numerical results to study the theoretical results. Superiority of the type II truncated Fréchet inverse exponential model over some known distributions is showed through one real data set.
\end{abstract}

Keywords: Type II truncated Fréchet, inverse exponential model, moments, simulation.

2010 MSC: 60E05, 62E15, 62F10.

(C)2020 All rights reserved.

\section{Introduction}

In the last years, many authors concerned with generated families of distributions. Some of the authors used the truncated models as the generators. Abid and Abdulrazak [1] used truncated Fréchet distribution on $(0,1)$ to get the truncated Fréchet-G family, Najarzadegan et al. [7] who proposed the truncated Weibull-G family by using the $(0,1)$ truncated Weibull model, Bantan et al. [4] who studied the truncated inverted Kumaraswamy $-G$ family by using the $(0,1)$ truncated inverted Kumaraswamy distribution. Recently, Aldahlan [2] has used $(0,1)$ truncated Fréchet distribution to get the type II truncated Fréchet generated family of distributions and it has the following distribution function (cdf) and probability density function (pdf)

$$
F(x ; b, \xi)=1-e e^{-(1-G(x ; \xi))^{-b}}, x \in R, \quad b>0 .
$$

and

$$
f(x ; b, \xi)=\operatorname{beg}(x ; \xi)(1-G(x ; \xi))^{-b-1} e^{-(1-G(x ; \xi))^{-b}} .
$$

If $X \sim \operatorname{exponential(E)}$ then the distribution of a random variable (r.v) $Y=1 / X$ follows an inverse expo-

Email address: maal-dahlan@uj .edu.sa (Maha A. Aldahlan)

doi: $10.22436 /$ jnsa.013.06.05

Received: 2019-11-24 Revised: 2020-02-06

Accepted: 2020-02-12 
nential (IE) model. The pdf and cdf of the IE model are

$$
g(x: \alpha)=\frac{\alpha}{x^{2}} e^{-\frac{\alpha}{x}}, \quad x, \alpha>0,
$$

and

$$
G(x: \alpha)=e^{-\frac{\alpha}{x}}, \quad x, \alpha>0 .
$$

The main objective here is to introduce the new model with two parameters which called type II truncated Fréchet inverse exponential (TIITFIE) distribution, study different statistical properties, maximum likelihood (ML) method is employed to estimate the population parameters of TIITFIE based on complete and censored samples.

By inserting (1.4) in (1.1) we get the cdf of TIITFIE model

$$
F(x ; b, \alpha)=1-e e^{-\left(1-e^{-\frac{\alpha}{x}}\right)^{-b}}, \quad x, b, \alpha>0 .
$$

The corresponding pdf to (1.5) is getting by inserting (1.3) and (1.4) in (1.2), then

$$
f(x ; b, \alpha)=\frac{\alpha b e}{x^{2}} e^{-\frac{\alpha}{x}}\left(1-e^{\left.-\frac{\alpha}{x}\right)^{-b-1}} e^{-\left(1-e^{-\frac{\alpha}{x}}\right)^{-b}}, \quad x, b, \alpha>0,\right.
$$

where $b$ is positive shape parameter and $\alpha$ is positive scale parameter.

Figures 1 and 2 show some pdf and cdf plots of $X \sim \operatorname{TIITFIE}(\omega=b, \alpha)$ for various parameter choices of $\omega$.
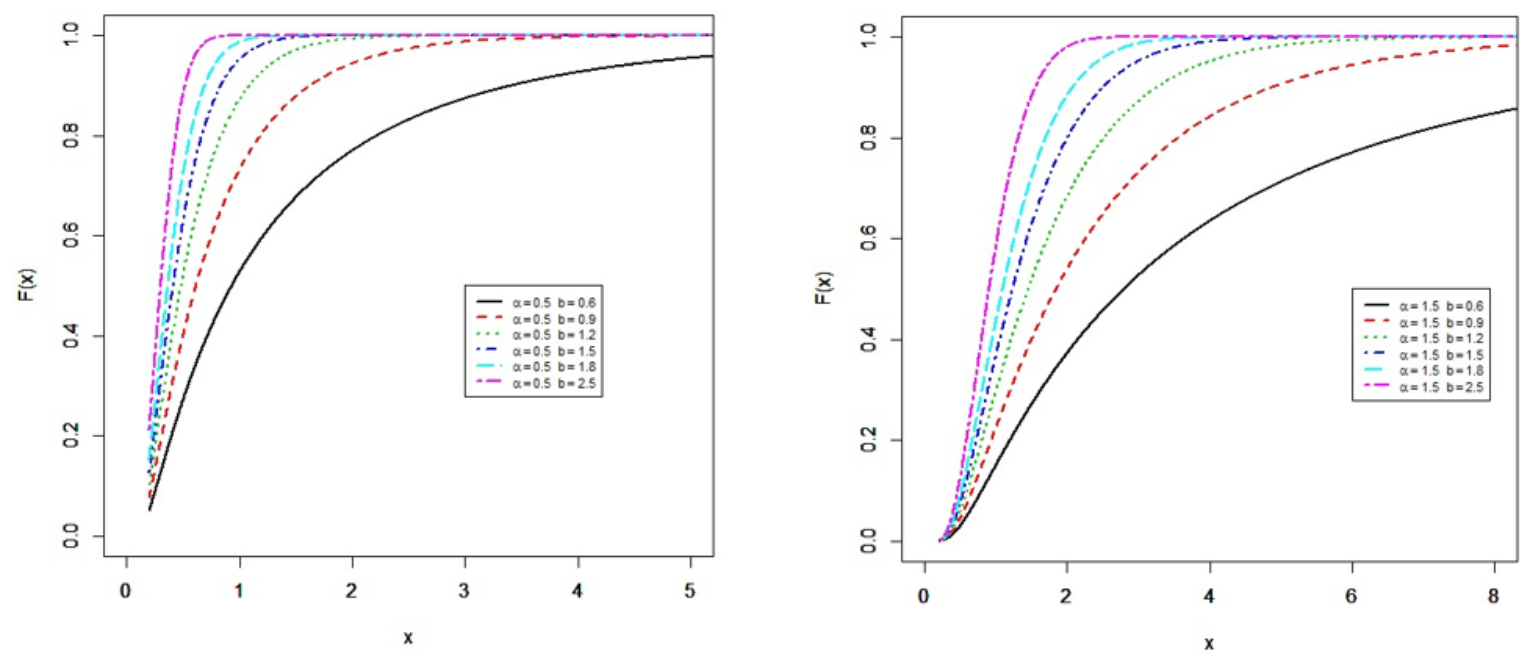

Figure 1: Plots of the cdf of the TIITFIE model.

The rest of this paper can be organized as follows. Sections 2 and 3 provide the reliability analysis and fundamental properties of the new model. In Section 4, point and approximate confidence interval (CI) of model Parameters are investigated under complete and Type II censoring (TIIC). In Section 5, the behavior of the estimates is studied via simulation. Application to real data set is analyzed to demonstrate the flexibility of TIITFIE model against some known models in Section 3. Finally, the paper ends with conclusion. 

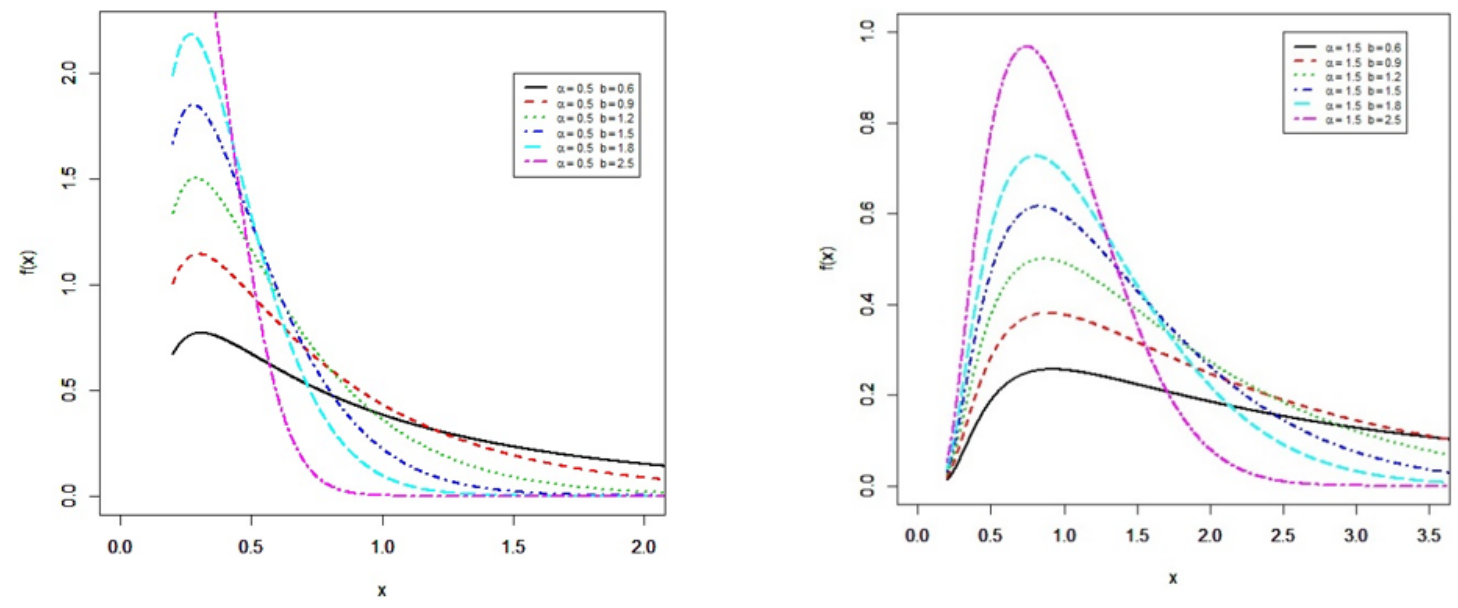

Figure 2: Plots of the pdf of the TIITFIE model.

\section{The reliability analysis for TIITFIE model}

The reliability function (rf), hazard rate function (hrf), reversed hrf and cumulative hrf of TIITFIE model are given by

$$
\begin{aligned}
& R(x ; b, \alpha)=e e^{-\left(1-e^{-\frac{\alpha}{x}}\right)^{-b}}, \\
& h(x ; b, \alpha)=\frac{\alpha b}{x^{2}} e^{-\frac{\alpha}{x}}\left(1-e^{\left.-\frac{\alpha}{x}\right)^{-b-1}},\right. \\
& \tau(x ; b, \alpha)=\frac{\frac{\alpha b e}{x^{2}} e^{-\frac{\alpha}{x}}\left(1-e^{\left.-\frac{\alpha}{x}\right)^{-b-1}} e^{-\left(1-e^{-\frac{\alpha}{x}}\right)^{-b}}\right.}{1-e e^{-\left(1-e^{-\frac{\alpha}{x}}\right)^{-b}}},
\end{aligned}
$$

and

$$
H(x ; b, \alpha)=\left(1-e^{-\frac{\alpha}{x}}\right)^{-b}-1 .
$$

Figures 3 and 4 shows some $\mathrm{rf}$ and hrf plots of $X \sim \operatorname{TIITFIE}(\omega=b, \alpha)$ for various parameter choices of $\omega$.
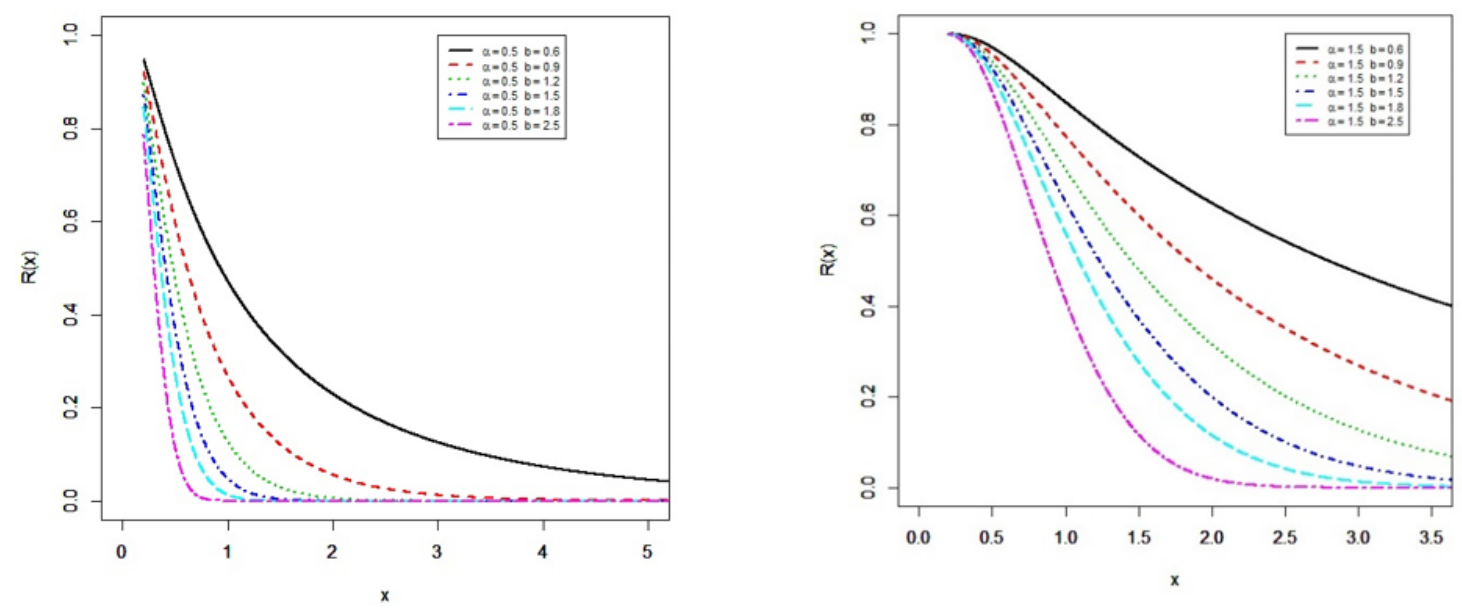

Figure 3: Plots of the rf of the TIITFIE model.

From Figures 2 and 4, we notice that the pdf of TIITFIE model can be uni-model, decreasing and right skewed. Also, the hrf of TIITFIE model can be increasing, constant and reversed J- shaped. 

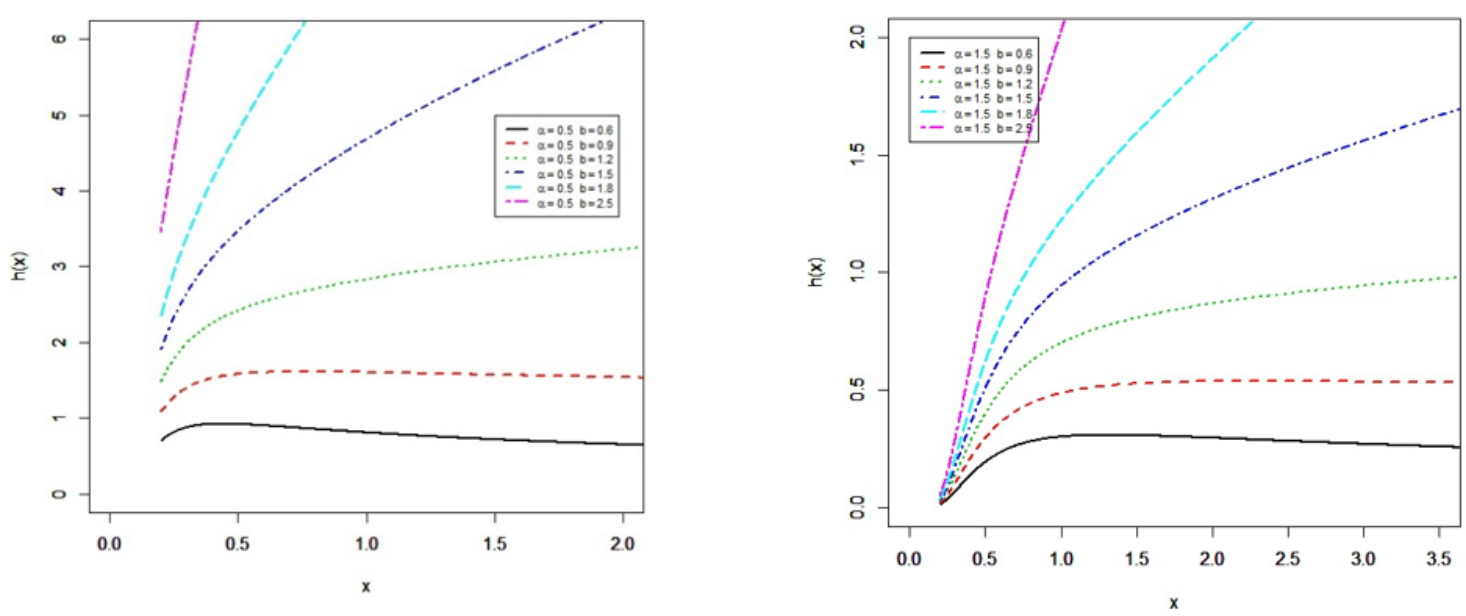

Figure 4: Plots of the hrf of the TIITFIE model.

\section{Some fundamental statistical properties}

In this section some fundamental statistical properties of the new model are derived.

3.1. Quantile and median

The quantile function, say $\mathrm{Q}(\mathrm{u})=\mathrm{F}^{-1}(\mathrm{u})$ of $\mathrm{X}$ is given by

$$
\mathrm{Q}_{(\mathfrak{u})}=\frac{-\alpha}{\ln \left\{1-\left[\ln \left(\frac{\mathrm{e}}{1-\mathfrak{u}}\right)\right]^{\frac{-1}{\mathrm{~b}}}\right\}},
$$

where, $u$ is a uniform $(0,1)$. The median $(M)$ can be calculated by putting $u=0.5$ in $(3.1)$, then

$$
M=\frac{-\alpha}{\ln \left\{1-[\ln (2 e)]^{\frac{-1}{b}}\right\}} .
$$

\subsection{Important expansion}

In this subsection important expansions of the pdf for TIITFIE model are proposed.

Aldahlan [2] rewrited the equation (1.2) as

$$
f(x)=\sum_{i=0}^{\infty} \eta_{i} g(x, \xi) G(x, \xi)^{i+1},
$$

where $\eta_{i}=\sum_{j=0}^{\infty} \frac{b e(-1)^{i+j}}{j !}\left(\begin{array}{c}b(j+1)+i \\ i\end{array}\right)$.

By substituting (1.3) and (1.4) in (3.2), then the pdf of TIITFIE distribution is

$$
f(x)=\sum_{i=0}^{\infty} \frac{w_{i}}{x^{2}} e^{-\frac{\alpha(i+2)}{x}},
$$

where $w_{k}=\alpha \eta_{i}$. 


\subsection{Moments}

If $\mathrm{X}$ has the pdf (3.3), then its $\mathrm{r}^{\text {th }}$ moment can be derived as

$$
\mu_{r}^{\prime}=E\left(X^{r}\right)=\int_{-\infty}^{\infty} x^{r} f(x ; \varphi) d x
$$

Inserting (3.3) into (3.4) yields:

$$
\mu_{r}^{\prime}=E\left(X^{r}\right)=\sum_{i=0}^{\infty} w_{i} \int_{0}^{\infty} x^{r-2} e^{-\alpha(i+2) x^{-1}} d x .
$$

Let $y=x^{-1}$, then

$$
\mu_{r}^{\prime}=\sum_{i=0}^{\infty} w_{i} \int_{0}^{\infty} y^{-r} e^{-\alpha(i+2) y} d x
$$

Then, $\mu_{r}^{\prime}$ becomes

$$
\mu_{r}^{\prime}=\sum_{i=0}^{\infty} \frac{w_{i} \Gamma(1-r)}{[\alpha(i+2)]^{1-r}}, \quad r<1 .
$$

The moment generating function of TIITFIE distribution is

$$
M_{X}(t)=\sum_{r=0}^{\infty} \frac{t^{r}}{r !} E\left(X^{r}\right)=\sum_{r, i=0}^{\infty} \frac{t^{r}}{r !} \frac{w_{i} \Gamma(1-r)}{[\alpha(i+2)]^{1-r}}, r<1
$$

The incomplete moments, say $\varphi_{s}(t)$, is

$$
\varphi_{s}(t)=\int_{0}^{t} x^{s} f(x ; \varphi) d x
$$

Using (3.2), then $\varphi_{s}(t)$ is given by

$$
\varphi_{s}(t)=\sum_{i=0}^{\infty} w_{i} \int_{0}^{t} x^{s-2} e^{-\alpha(i+2) x^{-1}} d x,
$$

then,

$$
\varphi_{s}(t)=\sum_{i=0}^{\infty} w_{k} \frac{v\left(1-s, \alpha(i+2) t^{-1}\right)}{(\alpha(i+2))^{1-s}}, s<1,
$$

where $v(s, t)=\int_{0}^{t} x^{s-1} e^{-x} d x$ is the lower incomplete gamma function.

Further, the conditional moments, say $\tau_{s}(t)$, is given by

$$
\tau_{s}(t)=\int_{t}^{\infty} x^{s} f(x ; \varphi) d x
$$

Hence, by using pdf (3.2), we can write

$$
\tau_{s}(t)=\sum_{i=0}^{\infty} w_{i} \int_{t}^{\infty} x^{s-2} e^{-\alpha(i+2) x^{-1}} d x,
$$

then,

$$
\tau_{s}(t)=\sum_{i=0}^{\infty} w_{i} \frac{\Gamma\left(1-s, \alpha(i+2) t^{-1}\right)}{(\alpha(i+2))^{1-s}}, s<1,
$$

where $\Gamma(s, t)=\int_{t}^{\infty} x^{s-1} e^{-x} d x$ is the upper incomplete gamma function. 


\section{ML estimation uder type II censored}

Let $X_{(1)}, \ldots, X_{(n)}$ be observed values from the TIITFIE model with set of parameters $\varphi=(\alpha, b)^{\top}$. The likelihood function under type II censored data can be expressed as

$$
L(\varphi)=K \prod_{i=1}^{r} f\left(x_{i}\right)\left[1-F\left(x_{r}\right)\right]^{n-r}
$$

where, and $K=\frac{n !}{(n-r) !}$ is constant and the total log-likelihood function under type II censored is

$$
\begin{aligned}
\ln L(\varphi)= & \ln \mathrm{K}+\mathrm{rln}(\alpha \mathrm{be})-2 \sum_{i=1}^{r} \ln x_{i}-\alpha \sum_{i=1}^{r} \frac{1}{x_{i}}-\sum_{i=1}^{r}\left(1-e^{\frac{-\alpha}{x_{i}}}\right)^{-b} \\
& -(b+1) \sum_{i=1}^{n} \ln \left(1-e^{\frac{-\alpha}{x_{i}}}\right)+n-r-(n-r)\left(1-e^{\frac{-\alpha}{x_{r}}}\right)^{-b} .
\end{aligned}
$$

The elements of the score function $\mathrm{U}_{\varphi}=\left(\mathrm{U}_{\alpha}, \mathrm{U}_{\mathrm{b}}\right)$ are given by

$$
u_{\alpha}=\frac{r}{\alpha}-\sum_{i=1}^{r} \frac{1}{x_{i}}+b \sum_{i=1}^{r} \frac{1}{x_{i}}\left(1-e^{\frac{-\alpha}{x_{i}}}\right)^{-b-1} e^{\frac{-\alpha}{x_{i}}}-(b+1) \sum_{i=1}^{r} \frac{\frac{1}{x_{i}} e^{\frac{-\alpha}{x_{i}}}}{1-e^{\frac{-\alpha}{x_{i}}}}+b(n-r) \frac{1}{x_{r}}\left(1-e^{\frac{-\alpha}{x_{r}}}\right)^{-b-1} e^{\frac{-\alpha}{x_{r}}}
$$

and

$$
\mathrm{u}_{\mathrm{b}}=\frac{\mathrm{r}}{\mathrm{b}}+\sum_{i=1}^{r}\left(1-e^{\frac{-\alpha}{x_{i}}}\right)^{-\mathrm{b}} \ln \left(1-e^{\frac{-\alpha}{x_{i}}}\right)-\sum_{i=1}^{r} \ln \left(1-e^{\frac{-\alpha}{x_{i}}}\right)+(n-r)\left(1-e^{\frac{-\alpha}{x_{r}}}\right)^{-b} \ln \left(1-e^{\frac{-\alpha}{x_{r}}}\right) .
$$

Then the ML estimators of the parameters $\alpha$ and $b$ are investigated by putting $\mathrm{U}_{\varphi}=0$ and solving them. An approximate CI of model parameters for TIITFIE model is obtained.

It is known that, the asymptotic distribution of ML estimates of elements of parameters $\alpha$ and $b$ is given by $((\hat{\alpha}-\alpha),(\hat{b}-b)) \rightarrow N\left(0, I^{-1}(\alpha, b)\right)$, where, $I^{-1}$ is the variance covariance matrix of unknown parameters, hence, the approximates $100(1-v) \%$ two sided CIs for $\alpha$ and $b$ are

$$
\hat{\alpha} \pm Z_{v / 2} \sqrt{\operatorname{var}(\hat{\alpha})}, \quad \hat{b} \pm Z_{v / 2} \sqrt{\operatorname{var}(\hat{b})}
$$

where, $Z_{v / 2}$ is the upper $v / 2^{\text {th }}$ percentile of the standard normal distribution.

\section{Numerical results}

This Section provides a numerical results to assess the behavior of the estimators in case of complete and TIIC. Mean square errors (MSEs), lower bound (LB) of CI and upper bound (UB) of CI, and average length (AL) of $90 \%$ and $95 \%$ are calculated via Mathematica 9. The following algorithm is designed as follows

1. 3000 random samples of size $n=30,50,100$, and 200 are generated from TIITFIE model.

2. Values of true parameters $(\alpha, b)$ are taken as $(0.5,0.5),(0.5,0.8)$, and $(0.8,0.8)$

3. Three levels of censoring are chosen as $r=60 \%, 80 \%$ (TIIC), and $100 \%$ (complete sample).

4. The ML estimates (MLEs), MSEs, LB, UB and AL for selected values of parameters are calculated.

5. Numerical outcomes are listed in Tables 1-3 based on complete and TIIC. 
Table 1: MLE, MSE, LB, UB and AL of TIITFIE model under TIIC for $(b=0.5, \alpha=0.5)$.

\begin{tabular}{|c|c|c|c|c|c|c|c|c|c|}
\hline \multirow{2}{*}{$n$} & \multirow[b]{2}{*}{$x_{r}$} & \multirow{2}{*}{ MLE } & \multirow{2}{*}{ MSE } & \multicolumn{3}{|c|}{$90 \%$} & \multicolumn{3}{|c|}{$95 \%$} \\
\hline & & & & LB & UB & $\mathrm{AL}$ & LB & UB & $\mathrm{AL}$ \\
\hline \multirow{6}{*}{30} & \multirow{2}{*}{$60 \%$} & 0.6054 & 0.0439 & 0.2877 & 0.9232 & 0.6355 & 0.2269 & 0.9840 & 0.7572 \\
\hline & & 0.6290 & 0.0805 & 0.2931 & 0.9649 & 0.6718 & 0.2288 & 1.0292 & 0.8005 \\
\hline & \multirow{2}{*}{$80 \%$} & 0.5886 & 0.0276 & 0.3620 & 0.8152 & 0.4533 & 0.3186 & 0.8586 & 0.5400 \\
\hline & & 0.6527 & 0.0654 & 0.3339 & 0.9715 & 0.6376 & 0.2728 & 1.0326 & 0.7597 \\
\hline & \multirow{2}{*}{$100 \%$} & 0.5244 & 0.0108 & 0.3797 & 0.6691 & 0.2894 & 0.3520 & 0.6968 & 0.3448 \\
\hline & & 0.5085 & 0.0147 & 0.2753 & 0.7417 & 0.4665 & 0.2306 & 0.7864 & 0.5558 \\
\hline \multirow{6}{*}{50} & \multirow{2}{*}{$60 \%$} & 0.6186 & 0.0248 & 0.3682 & 0.8691 & 0.5009 & 0.3202 & 0.9171 & 0.5969 \\
\hline & & 0.6282 & 0.0209 & 0.3674 & 0.8889 & 0.5215 & 0.3175 & 0.9388 & 0.6213 \\
\hline & \multirow{2}{*}{$80 \%$} & 0.5477 & 0.0092 & 0.3896 & 0.7057 & 0.3161 & 0.3593 & 0.7360 & 0.3767 \\
\hline & & 0.5808 & 0.0318 & 0.3574 & 0.8042 & 0.4468 & 0.3146 & 0.8470 & 0.5324 \\
\hline & \multirow{2}{*}{$100 \%$} & 0.5441 & 0.0067 & 0.4257 & 0.6624 & 0.2367 & 0.4031 & 0.6851 & 0.2820 \\
\hline & & 0.5325 & 0.0121 & 0.3442 & 0.7208 & 0.3766 & 0.3081 & 0.7569 & 0.4487 \\
\hline \multirow{6}{*}{100} & \multirow{2}{*}{$60 \%$} & 0.5464 & 0.0110 & 0.3959 & 0.6969 & 0.3010 & 0.3671 & 0.7257 & 0.3586 \\
\hline & & 0.5711 & 0.0190 & 0.3996 & 0.7425 & 0.3430 & 0.3667 & 0.7754 & 0.4086 \\
\hline & \multirow{2}{*}{$80 \%$} & 0.5336 & 0.0069 & 0.4246 & 0.6427 & 0.2181 & 0.4037 & 0.6635 & 0.2598 \\
\hline & & 0.5133 & 0.0062 & 0.3723 & 0.6543 & 0.2821 & 0.3453 & 0.6813 & 0.3361 \\
\hline & \multirow{2}{*}{$100 \%$} & 0.4990 & 0.0021 & 0.4239 & 0.5741 & 0.1502 & 0.4095 & 0.5885 & 0.1790 \\
\hline & & 0.4868 & 0.0069 & 0.3617 & 0.6120 & 0.2503 & 0.3377 & 0.6360 & 0.2982 \\
\hline \multirow{6}{*}{200} & \multirow{2}{*}{$60 \%$} & 0.5231 & 0.0033 & 0.4234 & 0.6228 & 0.1995 & 0.4043 & 0.6419 & 0.2376 \\
\hline & & 0.5063 & 0.0048 & 0.3979 & 0.6147 & 0.2168 & 0.3771 & 0.6355 & 0.2584 \\
\hline & \multirow{2}{*}{$80 \%$} & 0.5026 & 0.0012 & 0.4320 & 0.5733 & 0.1413 & 0.4184 & 0.5868 & 0.1684 \\
\hline & & 0.4916 & 0.0025 & 0.3946 & 0.5886 & 0.1940 & 0.3760 & 0.6072 & 0.2312 \\
\hline & \multirow{2}{*}{$100 \%$} & 0.5067 & 0.0014 & 0.4537 & 0.5597 & 0.1060 & 0.4435 & 0.5699 & 0.1264 \\
\hline & & 0.5077 & 0.0040 & 0.4161 & 0.5993 & 0.1831 & 0.3986 & 0.6168 & 0.2182 \\
\hline
\end{tabular}

Table 2: MLE, MSE, LB, UB and AL of TIITFIE model under TIIC for $(b=0.8, \alpha=0.5)$.

\begin{tabular}{|c|c|c|c|c|c|c|c|c|c|}
\hline \multirow[b]{2}{*}{$n$} & \multirow[b]{2}{*}{$x_{r}$} & \multirow{2}{*}{ MLE } & \multirow{2}{*}{ MSE } & \multicolumn{3}{|c|}{$90 \%$} & \multicolumn{3}{|c|}{$95 \%$} \\
\hline & & & & LB & UB & $\mathrm{AL}$ & LB & UB & AL \\
\hline \multirow{6}{*}{30} & \multirow{2}{*}{$60 \%$} & 1.2138 & 0.4326 & 0.4004 & 2.0272 & 1.6269 & 0.2446 & 2.1830 & 1.9384 \\
\hline & & 0.6448 & 0.0454 & 0.3366 & 0.9529 & 0.6163 & 0.2776 & 1.0120 & 0.7344 \\
\hline & \multirow{2}{*}{$80 \%$} & 0.8859 & 0.1501 & 0.4880 & 1.2838 & 0.7958 & 0.4119 & 1.3600 & 0.9481 \\
\hline & & 0.5101 & 0.0237 & 0.2824 & 0.7379 & 0.4554 & 0.2388 & 0.7815 & 0.5426 \\
\hline & \multirow{2}{*}{$100 \%$} & 0.8573 & 0.0356 & 0.5788 & 1.1358 & 0.5569 & 0.5255 & 1.1891 & 0.6636 \\
\hline & & 0.5075 & 0.0184 & 0.3038 & 0.7113 & 0.4075 & 0.2648 & 0.7503 & 0.4855 \\
\hline \multirow{6}{*}{50} & \multirow{2}{*}{$60 \%$} & 1.0009 & 0.1827 & 0.5206 & 1.4811 & 0.9606 & 0.4286 & 1.5731 & 1.1445 \\
\hline & & 0.5938 & 0.0267 & 0.3688 & 0.8188 & 0.4500 & 0.3257 & 0.8619 & 0.5362 \\
\hline & \multirow{2}{*}{$80 \%$} & 0.8710 & 0.0340 & 0.5777 & 1.1644 & 0.5866 & 0.5216 & 1.2205 & 0.6989 \\
\hline & & 0.5540 & 0.0180 & 0.3629 & 0.7451 & 0.3823 & 0.3263 & 0.7817 & 0.4555 \\
\hline & \multirow{2}{*}{$100 \%$} & 0.8714 & 0.0159 & 0.6519 & 1.0910 & 0.4391 & 0.6098 & 1.1330 & 0.5232 \\
\hline & & 0.5408 & 0.0078 & 0.3732 & 0.7084 & 0.3352 & 0.3411 & 0.7405 & 0.3994 \\
\hline \multirow{6}{*}{100} & \multirow{2}{*}{$60 \%$} & 0.9169 & 0.0371 & 0.6189 & 1.2149 & 0.5961 & 0.5618 & 1.2720 & 0.7102 \\
\hline & & 0.5607 & 0.0114 & 0.4085 & 0.7129 & 0.3044 & 0.3794 & 0.7420 & 0.3627 \\
\hline & \multirow{2}{*}{$80 \%$} & 0.8567 & 0.0184 & 0.6526 & 1.0608 & 0.4083 & 0.6135 & 1.0999 & 0.4865 \\
\hline & & 0.5368 & 0.0050 & 0.4046 & 0.6691 & 0.2645 & 0.3793 & 0.6944 & 0.3151 \\
\hline & \multirow{2}{*}{$100 \%$} & 0.8081 & 0.0049 & 0.6695 & 0.9466 & 0.2771 & 0.6430 & 0.9731 & 0.3301 \\
\hline & & 0.5123 & 0.0054 & 0.3985 & 0.6261 & 0.2276 & 0.3767 & 0.6479 & 0.2712 \\
\hline \multirow{6}{*}{200} & \multirow{2}{*}{$60 \%$} & 0.7935 & 0.0050 & 0.6204 & 0.9665 & 0.3462 & 0.5872 & 0.9997 & 0.4124 \\
\hline & & 0.4931 & 0.0021 & 0.3961 & 0.5902 & 0.1941 & 0.3775 & 0.6087 & 0.2312 \\
\hline & \multirow{2}{*}{$80 \%$} & 0.8203 & 0.0048 & 0.6854 & 0.9553 & 0.2698 & 0.6596 & 0.9811 & 0.3215 \\
\hline & & 0.5188 & 0.0022 & 0.4280 & 0.6096 & 0.1816 & 0.4106 & 0.6270 & 0.2164 \\
\hline & \multirow{2}{*}{$100 \%$} & 0.8071 & 0.0012 & 0.7090 & 0.9051 & 0.1961 & 0.6902 & 0.9239 & 0.2337 \\
\hline & & 0.4929 & 0.0013 & 0.4153 & 0.5704 & 0.1551 & 0.4004 & 0.5853 & 0.1848 \\
\hline
\end{tabular}


Table 3: MLE, MSE, LB, UB and AL of TIITFIE model under TIIC for $(b=0.8, \alpha=0.8)$.

\begin{tabular}{|c|c|c|c|c|c|c|c|c|c|}
\hline \multirow{2}{*}{$n$} & \multirow[b]{2}{*}{$x_{r}$} & \multirow{2}{*}{ MLE } & \multirow{2}{*}{ MSE } & \multicolumn{3}{|c|}{$90 \%$} & \multicolumn{3}{|c|}{$95 \%$} \\
\hline & & & & LB & UB & $\mathrm{AL}$ & LB & UB & $\mathrm{AL}$ \\
\hline \multirow{8}{*}{30} & \multirow{2}{*}{$60 \%$} & 1.0354 & 0.5166 & 0.3617 & 1.7090 & 1.3472 & 0.2328 & 1.8380 & 1.6052 \\
\hline & & 0.9628 & 0.2372 & 0.4951 & 1.4305 & 0.9353 & 0.4056 & 1.5200 & 1.1145 \\
\hline & \multirow{2}{*}{$80 \%$} & 1.0058 & 0.1469 & 0.5360 & 1.4757 & 0.9398 & 0.4460 & 1.5657 & 1.1197 \\
\hline & & 0.9196 & 0.0685 & 0.5187 & 1.3205 & 0.8018 & 0.4419 & 1.3973 & 0.9553 \\
\hline & \multirow{2}{*}{$100 \%$} & 0.9269 & 0.0685 & 0.6157 & 1.2382 & 0.6225 & 0.5561 & 1.2978 & 0.7417 \\
\hline & & 0.9290 & 0.0781 & 0.5623 & 1.2956 & 0.7333 & 0.4921 & 1.3658 & 0.8737 \\
\hline & \multirow{2}{*}{$60 \%$} & 1.0652 & 0.1509 & 0.5494 & 1.5809 & 1.0315 & 0.4507 & 1.6796 & 1.2290 \\
\hline & & 0.9990 & 0.0566 & 0.6241 & 1.3738 & 0.7497 & 0.5524 & 1.4456 & 0.8932 \\
\hline \multirow{6}{*}{50} & \multirow{2}{*}{$80 \%$} & 0.8997 & 0.0383 & 0.5939 & 1.2055 & 0.6116 & 0.5353 & 1.2640 & 0.7287 \\
\hline & & 0.8474 & 0.0271 & 0.5566 & 1.1383 & 0.5817 & 0.5009 & 1.1940 & 0.6931 \\
\hline & \multirow{2}{*}{$100 \%$} & 0.7864 & 0.0156 & 0.5973 & 0.9754 & 0.3780 & 0.5612 & 1.0116 & 0.4504 \\
\hline & & 0.8063 & 0.0296 & 0.5512 & 1.0615 & 0.5103 & 0.5023 & 1.1104 & 0.6080 \\
\hline & \multirow{2}{*}{$60 \%$} & 0.9009 & 0.0517 & 0.6082 & 1.1936 & 0.5854 & 0.5522 & 1.2496 & 0.6974 \\
\hline & & 0.8185 & 0.0411 & 0.5959 & 1.0411 & 0.4452 & 0.5533 & 1.0837 & 0.5304 \\
\hline \multirow{4}{*}{100} & \multirow[b]{2}{*}{80} & 0.7873 & 0.0178 & 0.6060 & 0.9685 & 0.3626 & 0.5713 & 1.0032 & 0.4320 \\
\hline & & 0.7534 & 0.0155 & 0.5651 & 0.9417 & 0.3766 & 0.5291 & 0.9778 & 0.4487 \\
\hline & \multirow{2}{*}{$100 \%$} & 0.8007 & 0.0074 & 0.6643 & 0.9372 & 0.2729 & 0.6382 & 0.9633 & 0.3251 \\
\hline & & 0.7762 & 0.0132 & 0.6034 & 0.9490 & 0.3456 & 0.5703 & 0.9821 & 0.4117 \\
\hline \multirow{6}{*}{200} & \multirow{2}{*}{$60 \%$} & 0.7784 & 0.0064 & 0.6097 & 0.9472 & 0.3375 & 0.5774 & 0.9795 & 0.4021 \\
\hline & & 0.7679 & 0.0051 & 0.6163 & 0.9195 & 0.3033 & 0.5872 & 0.9485 & 0.3613 \\
\hline & \multirow{2}{*}{$80 \%$} & 0.8334 & 0.0102 & 0.6953 & 0.9714 & 0.2762 & 0.6688 & 0.9979 & 0.3291 \\
\hline & & 0.8284 & 0.0095 & 0.6838 & 0.9730 & 0.2892 & 0.6561 & 1.0007 & 0.3446 \\
\hline & \multirow{2}{*}{$100 \%$} & 0.7870 & 0.0034 & 0.6926 & 0.8814 & 0.1888 & 0.6746 & 0.8995 & 0.2250 \\
\hline & & 0.7876 & 0.0057 & 0.6630 & 0.9123 & 0.2493 & 0.6391 & 0.9361 & 0.2971 \\
\hline
\end{tabular}

It is noticed from Tables 1-3 that the MSEs, LB, UB and AL are decreased when $n$ is increased and when level of censoring is increased.

\section{Application}

Here, we fit the TIITFIE model to one real data. This data is proposed by Crowder et al. [5] contains the ball bearing data, which indicates the number of revolutions before failure for ball bearing.

We compare the fits of the TIITFIE model with some known models like; the beta IE (BIE) model (see Khan [6]) by keeping shape parameter is equal to one, logistic IE (LIE) model (see Oguntunde et al. [8]), inverse Weibull inverse exponential (IWIE) model (see Aldahlan [3]), and standard IE model.

We estimate the model Parameterss by using ML method. We compare the goodness-of-fit of the models using negative two $\log \mathrm{L}(-2 \log \mathrm{L})$, Akaike information criterion (AIC), corrected Akaike information criterion (CAIC), Bayesian information criterion (BIC), Hannan Quinn information criterion (HQIC), Anderson Darling statistic $\left(\mathrm{A}^{*}\right)$ and Cramér-von Mises statistic $\left(\mathrm{W}^{*}\right)$.

Table 4: MLEs, SEs and measures of goodness-of-fit statistics for the data set.

\begin{tabular}{|l|c|l|l|l|l|l|l|l|l|l|}
\hline Models & \multicolumn{2}{|c|}{ MLEs and (SEs) } & -2LogL & AIC & CAIC & BIC & HQIC & $\mathrm{A}^{*}$ & $\mathrm{~W}^{*}$ \\
\hline TIITFIE $(\boldsymbol{\alpha}, \mathrm{b})$ & $\begin{array}{c}94.631 \\
(21.514)\end{array}$ & $\begin{array}{l}1.945 \\
(0.545)\end{array}$ & - & 234.911 & 238.911 & 239.482 & 237.671 & 239.536 & 0.238 & 0.041 \\
\hline BIE $(\theta, \mathrm{a}, \mathrm{b})$ & $\begin{array}{c}11.722 \\
(0.131)\end{array}$ & $\begin{array}{l}2.825 \\
(0.268)\end{array}$ & $\begin{array}{l}1.667 \\
(0.953)\end{array}$ & 237.43 & 243.43 & 244.63 & 241.571 & 244.368 & 0.357 & 0.05 \\
\hline LIE $(\mathrm{a}, \mathrm{b})$ & $\begin{array}{c}2.459 \\
(0.279)\end{array}$ & $\begin{array}{l}1.238 \\
(1.226)\end{array}$ & - & 238.279 & 242.279 & 242.85 & 241.039 & 242.904 & 0.375 & 0 \\
\hline $\operatorname{IWIE}(\boldsymbol{\alpha}, \boldsymbol{\beta}, \boldsymbol{\theta})$ & $\begin{array}{c}1.247 \\
(2.115)\end{array}$ & $\begin{array}{l}1.281 \\
(0.144)\end{array}$ & $\begin{array}{l}14.33 \\
(5.374)\end{array}$ & 241.346 & 247.346 & 248.546 & 245.487 & 248.284 & 0.640 & 0.091 \\
\hline $\operatorname{IE}(\theta)$ & $\begin{array}{c}20.403 \\
(2.55)\end{array}$ & - & - & 253.927 & 255.927 & & & & 0.356 & 0.0498 \\
\hline
\end{tabular}


The MLEs of the competitive models and their corresponding standard errors (SEs) are listed in Table 4 also, the values of AIC, CAIC, BIC, HQIC, $\mathrm{A}^{*}$ and $\mathrm{W}^{*}$ are showed in the same table.

Table 4 shows that the TIITFIE model has the lowest values for AIC, CAIC, BIC, HQIC, $\mathrm{A}^{*}$ and $\mathrm{W}^{*}$ among all fitted models. So, the TIITFIE is the best model. The estimated pdf, pp plot, cdf and rf for the TIITFIE model are showed in Figures 5 and 6.
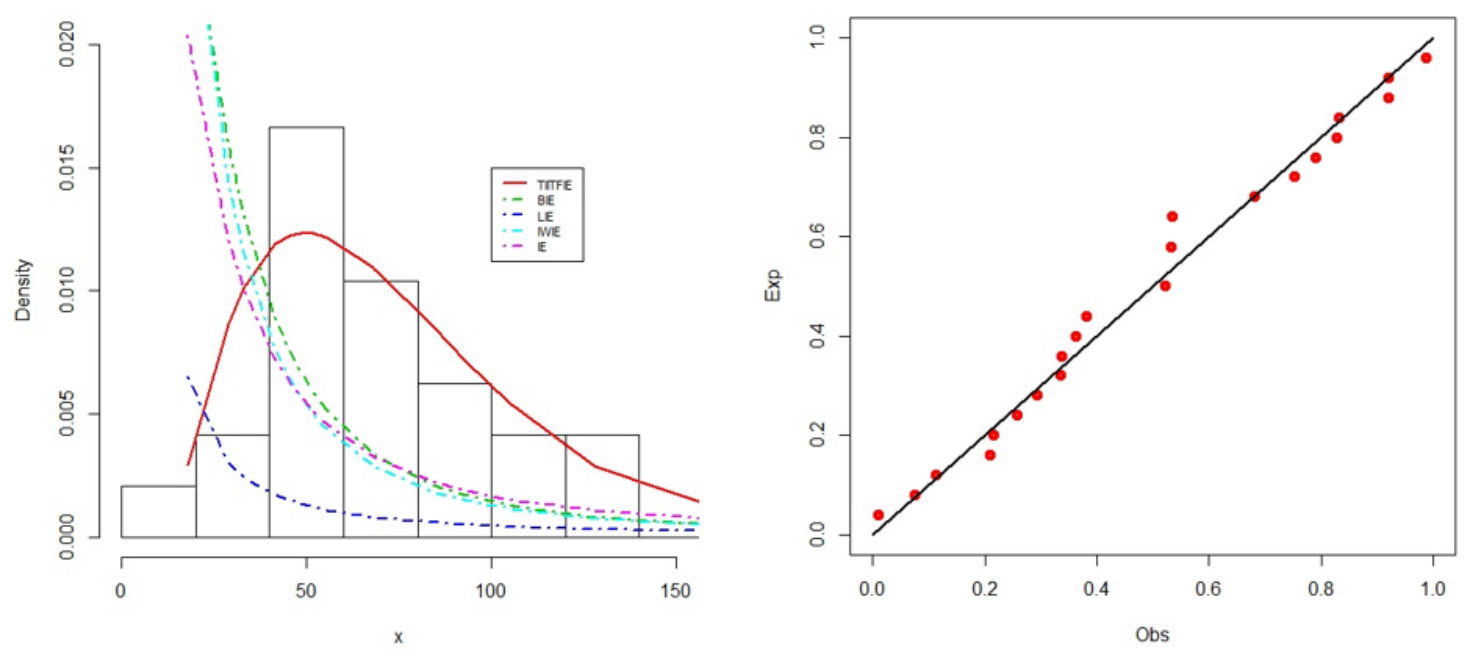

Figure 5: The estimated pdf and pp plot of the TIITFIE model.
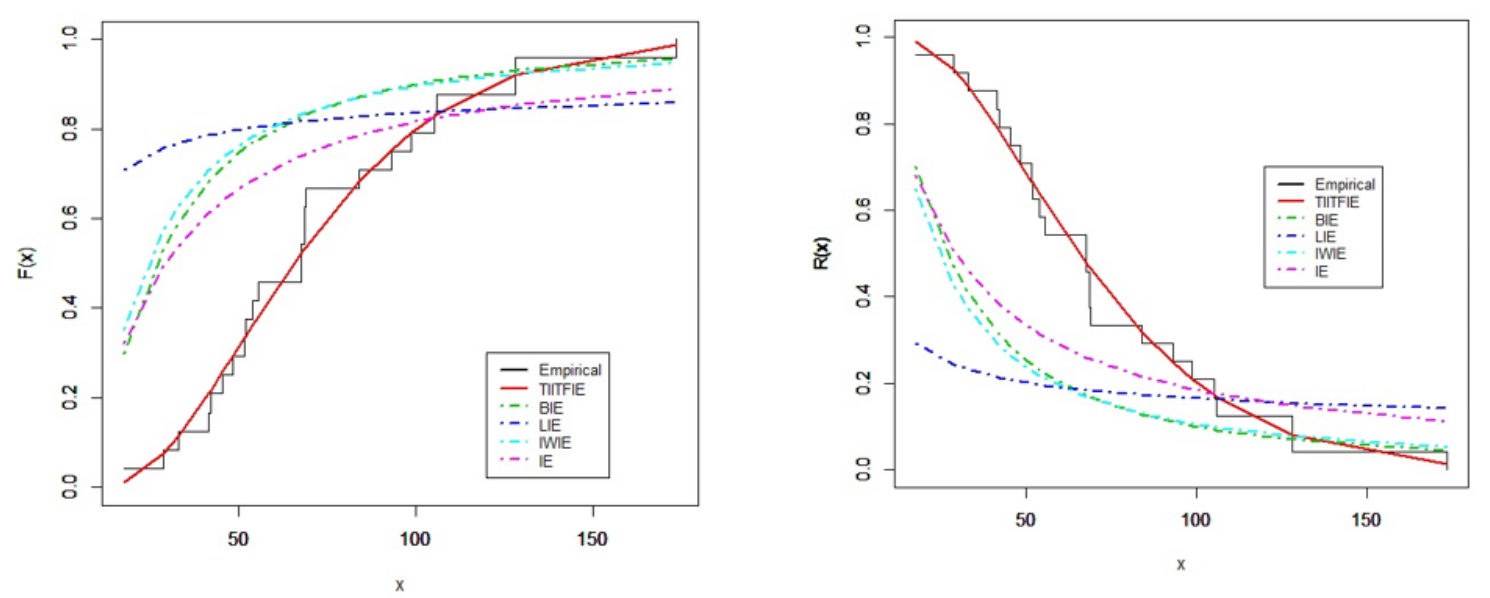

Figure 6: The estimated cdf and rf of the TIITFIE model.

\section{Conclusions}

A new probability distribution related to IE model, the so called TIITFIE distribution is proposed. Moments, incomplete moments, conditional moments and quantile function of the new model are calculated. Parameter estimation of model parameters is discussed via maximum likelihood method from complete and censored samples. Also, asymptotic confidence intervals of parameters are computed. Good performance and accuracy of the maximum likelihood estimators of the parameters are examined through a numerical results. Application to one real data set show that the fit of the new model is superior to some other known models. 


\section{References}

[1] S. H. Abid, R. K. Abdulrazak, Truncated Fréchet-G generator of distributions, Appl. Math., 7 (2017), 51-66. 1

[2] M. A. Aldahlan, Type II Truncated Fréchet Generated Family of Distributions, Int. J. Math. Appl., 7 (2019), 221-228. 1, 3.2

[3] M. A. Aldahlan, The inverse Weibull inverse exponential distribution with application, Int. J. Contemp. Math. Sci., 14 (2019), 17-30. 6

[4] R. A. R. Bantan, F. Jamal, C. Chesneau, M. Elgarhy, Truncated inverted Kumaraswamy generated family of distributions with applications, Entropy, 21 (2019), 1-22. 1

[5] M. J. Crowder, A. C. Kimber, R. L. Smith, T. J. Sweeting, Statistical Analysis of Reliability Data, Chapman and Hall, London, (1991). 6

[6] M. S. Khan, The beta inverse Weibull distribution, Int. Trans. Math. Sci. Comput., 3 (2010), 113-119. 6

[7] H. Najarzadegan, M. H. Alamatsaz, S. Hayati, Truncated Weibull-G more flexible and more reliable thangeta-G distribution, Int. J. Stat. Probab., 6 (2017), 1-17. 1

[8] P. E. Oguntunde, A. O. Adejumo, M. A. Khaleel, H. I. Okagbue, O. A. Odetunmibi, The logistic inverse exponential distribution: Basic structural properties and application, In Proceedings of the World Congress on Engineering and Computer Science (San Francisco, U.S.A.), 2018 (2018), 4 pages. 6 